\title{
The perioperative application of High Flow Nasal Cannula: a single center experience
}

\begin{abstract}
The physiologic benefits of high-flow nasal cannula (HFNC) therapy are well documented in the intensive care literature, yet only a handful of case reports describe its perioperative use. Our single center experience explored comprehensive applications of HFNC in the perioperative setting. Over a ten-month period, HFNC was used in the care of 85 medically complex patients either as an adjuvant or main oxygen therapy during induction of general anesthesia, maintenance of deep intraoperative sedation, and during early postoperative care. Here, we illustrate clinical scenarios in which HFNC therapy made patient care safer and describe a framework for integrating this technology into the anesthesia practice at our institution.
\end{abstract}

Volume I 2 Issue I - 2020

\author{
Philip C. Carullo,' Dennis P. Phillips, ${ }^{2}$ \\ Ezeldeen Abuelkasem, ${ }^{3}$ Ibtesam A. Hilmi ${ }^{4}$ \\ 'University of Pittsburgh Medical Center, Children's Hospital \\ of Pittsburgh, Department of Anesthesiology and Perioperative \\ Medicine, USA \\ ${ }^{2}$ University of Pittsburgh Medical Center, Director of the \\ Quality Improvement, Department of Anesthesiology and \\ Perioperative Medicine, USA \\ ${ }^{3}$ University of Pittsburgh Medical Center, Department of \\ Anesthesiology and Perioperative Medicine, USA \\ ${ }^{4}$ Professor, Department of Anesthesiology and Perioperative \\ Medicine, University of Pittsburgh Medical Center Presbyterian \\ Hospital, USA
}

\begin{abstract}
Correspondence: Ibtesam A. Hilmi, MBCHB, FRCA, Professor Department of Anesthesiology and Perioperative Medicine, University of Pittsburgh Medical Center Presbyterian Hospital, 200 Lothrop St, Anesthesia office, C-wing, suite 204, Pittsburgh, PA I52I3, USA, Tel 4I2-647-3262, Fax 4|2-647-6290, Email hilmiia@anes.upmc.edu
\end{abstract}

Received: January 03, 2020 | Published: January 13, 2020

\section{Introduction}

High-flow nasal cannula (HFNC) therapy has been used in a variety of clinical scenarios in recent years, particularly in the intensive care unit (ICU) setting. The HFNC system provides heated and humidified oxygen at flows much higher than standard oxygen modalities (up to 70 liters per minute) and it is well-tolerated by patients. The high flow provides several physiologic benefits with promising utilization in the perioperative setting. These benefits include reduction of the ApneaHypopnea Index in patients with obstructive sleep apnea (OSA) by $64 \%$, with flows greater than $20 \mathrm{lpm}$ and nasally-generated positive end-expiratory pressure (PEEP) of 3-5 $\mathrm{mmHg}$ at flows greater than $35 \mathrm{lpm} .{ }^{1,2}$ Unlike traditional non-invasive ventilation approaches such as bilevel positive pressure (BiPAP), these benefits are created from the high flow of gas, therefore allowing airway manipulation with prevention of apnea and hypoxia and preservation of PEEP effects.

In several randomized controlled trials and systematic literature reviews, HFNC was not shown to be inferior to BiPAP at preventing intubation in patients with acute respiratory failure and/or reintubation in a subset of post-surgical patients..$^{3-6}$ In 2015, Patel and Nouraei published their landmark article on Transnasal Humidified Rapid-Insufflation Ventilatory Exchange (THRIVE), where HFNC increased apnea time in 25 patients with difficult airways undergoing laryngeal surgery. The average apnea time was 17 minutes and no patient experienced desaturation $<90 \%{ }^{7}$ The use of HFNC in difficult airway management is being discussed by major international airway societies, with several countries already incorporating it into their difficult airway management algorithms. ${ }^{8}$

Airway obstruction and apnea with resultant hypoxia can occur throughout a patient's perioperative course, so it is not surprising to see a surge in case reports illustrating the benefit of HFNC during intraoperative patient care. ${ }^{9-11}$ In this report, we describe a framework for integrating HFNC therapy into anesthesia practice and illustrate key clinical scenarios where its use results in better and safer patient care.

\section{Methods}

This project aimed to improve patient care and educate and train anesthesia providers about the use and applications of HFNC in the perioperative setting for certain patients and/or surgical procedures. Since the study was not meant to be a scientific research protocol, it was open-label, unblended, and had no case-matched control for comparison. We relied on providers' experience and feedback regarding HFNC. This project was conducted over a ten-month period at UPMC (University of Pittsburgh Medical Center) Presbyterian Hospital, a large quaternary-care academic healthcare center. The protocol was approved by the hospital's Quality Improvement (QI) Committee as a QI project, and the requirement for written informed patient consent was waived. HFNC was first introduced to the Department of Anesthesiology and Perioperative Medicine at a patient safety meeting. This meeting included a presentation of the literature, a description of the device, and suggested clinical scenarios for use. This was followed by a day of in-service training.

The HFNC machine used in this project was the Fisher and Paykel $\mathrm{AIRVO}_{2}$, which required a standard three-pronged power cord and piped oxygen. Quick-Connect Ohmeda and Chemetron adapters were used to accommodate the various oxygen outlets in the operating rooms (ORs) and post-anesthesia care unit (PACU). Five units were obtained for our 47 main ORs, stored in the anesthesia workroom, and were immediately available for use. Fisher and Paykel provided the 
$\mathrm{AIRVO}_{2}$ units for the project; however, no other financial relationships or incentives were required or agreed upon by the investigators.

Anesthesia technologists were trained to properly clean and adequately sterilize the machine after its use. Anesthesia providers would simply notify the anesthesia technologist of anticipated HFNC use, and technologist would bring the HFNC unit to the OR and have it ready for use upon the patient's arrival. In the PACU, respiratory therapists were in charge of setting up the device according to the anesthesia team's recommendations.

The investigators recommended HFNC settings for the following perioperative scenarios:

1. Induction of general anesthesia: recommended settings $\mathrm{FiO}_{2}$ $100 \%$, rate 40-60 lpm.

2. Maintenance of anesthesia: recommended settings $\mathrm{FiO}_{2} 30$ $100 \%$, rate $40 \mathrm{lpm}$

3. Post-anesthesia care: recommended settings $\mathrm{FiO}_{2} 100 \%$, rate 40 lpm

\section{Inclusion criteria:}

i. Patients undergoing elective or emergent awake tracheostomy for severely compromised airway (tumors, airway edema, infection, and trauma).

ii. Endoscopic esophageal dilatation under deep sedation and endoscopic retrograde ancreaticoduodenectomy.

iii. Awake fiberoptic intubation in patients with compromised airway or known difficult intubation.

iv. Surgical procedures under deep sedation for patients with known risk for airway obstruction, OSA, and/or morbid obesity.

v. In patients undergoing live-donor liver transplantation where the anesthesia team is planning for early extubation (within one hour of completion of the procedure).

vi. In patients during their PACU stay suffering from airway obstruction or hypoxia that does not respond to the usual measures (face mask $\mathrm{O}_{2}$ therapy) in an attempt to prevent reintubation. Also, in patients who are well-known to present difficult intubation and those with OSA, morbid obesity, and serious respiratory disease (chronic obstructive pulmonary disease (COPD), emphysema, respiratory compromise).

The anesthesia team enrolled patients in accordance with the selection criteria stated in the QI project. Although the investigators set the flow rate and $\mathrm{FiO}_{2}$, anesthesia attendings were able to change the setup according to the patient's requirements. However, providers followed American Society of Anesthesiologists Fire Safety Guidelines to avoid $\mathrm{FiO}_{2}>30 \%$ in cases above spinal vertebra T5 where either electrocautery or laser therapy use was anticipated.

Anesthesia attendings reported data to the investigative team, including the following information: indication for HFNC use, when it was used (induction of anesthesia, during maintenance or postoperative period), patient satisfaction, provider satisfaction, whether the goals were achieved by maintaining patent airway and satisfactory oxygenation, and any complications or safety-related concerns.

\section{Results}

Over the course of ten months, HFNC therapy was used in the care of 85 patients. The average patient age was 62 years old and the average flow rate and $\mathrm{FiO}_{2}$ were $35 \mathrm{lpm}$ and $60 \%$, respectively. No complications were reported, but providers did report difficulty obtaining end-tidal $\mathrm{CO}_{2}$ and potential fire hazards as safety concerns (see Intraoperative Management).

Figure 1 depicts where and how often HFNC was used in the perioperative setting. HFNC therapy was used in 22 patients' induction, defined as taking place in the OR during the patient's initial airway management. HFNC was used in 40 patients' intraoperative management, defined as occurring during sedations or endotracheal tube exchange in the maintenance phase of an anesthetic. Twentythree patients were given postoperative HFNC therapy, defined as occurring outside of the OR and under the supervision of a PACU or ICU nurse.

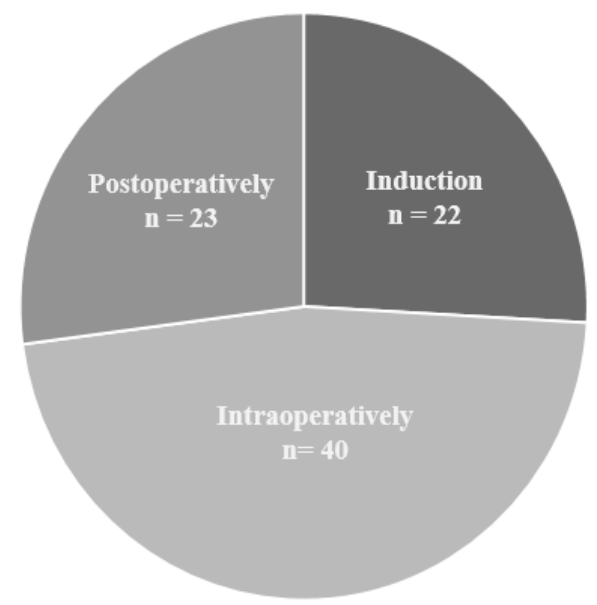

Figure I Perioperative use of High Flow Nasal Cannula (HFNC):Where was it used?

Graphic describing where HFNC was used in the perioperative setting. HFNC was used in the perioperative care of 85 patients. Induction use was defined as in the operating room (OR) during the initial airway management of the patient. Intraoperative use was defined as during a sedation or endotracheal tube exchange in the maintenance phase of an anesthetic or at emergence following extubation in the OR. Postoperative use was defined as occurring outside of the operating suite and under the supervision of a PACU or ICU nurse.

\section{Induction of AnesthesialAirway Management: $\mathrm{n}=\mathbf{2 2}$ patients}

HFNC was most often used as the main oxygen therapy during awake fiberoptic intubations. These patients presented with risk factors for difficult intubation, including comorbidities such as acute stridor, laryngeal mass, neck lymphedema or tracheomalacia, history of difficult intubation, unstable cervical spine, and super morbid obesity. Awake tracheostomy, endotracheal tube exchanges, and apneic oxygenation during endotracheal tube placement were also common indications for HFNC use.

Table 1 (Induction of Anesthesia section) illustrates key scenarios in which HFNC was considered to provide safer oxygen therapy during 
induction of anesthesia compared to conventional oxygen therapies. These medically complex patients were vulnerable to hypoxia, desaturation, and cardiovascular collapse during this time; however, they all underwent induction without airway-related complications.

\section{Intraoperative management: $\mathrm{n}=40$ patients}

HFNC therapy was used intraoperatively in 40 patients, most often during monitored sedations in patients with OSA, morbid obesity, or $\mathrm{O}_{2}$-dependent lung disease. Procedures requiring sedation included esophagogastroduodenoscopies, hernia repairs, deep brain stimulator and implantable cardioverter defibrillator/pacemaker placement, nerve biopsies, angiography, infusaport placement, and limb amputations. HFNC therapy was also used in several endotracheal and tracheostomy tube exchanges under general anesthesia.

Table I Illustrative Cases Where HFNC was felt to Provide Safer Oxygen Therapy in the Perioperative Setting Compared to Conventional Oxygen Therapies

\begin{tabular}{|c|c|c|c|c|}
\hline \multicolumn{5}{|c|}{ Induction of Anesthesia } \\
\hline Age & HPI / Comorbid Disease & Procedure & BMI $\left(\mathbf{k g} / \mathbf{m}^{2}\right)$ & Miscellaneous \\
\hline 56 & $\begin{array}{l}\text { HTN, HFpEF, } \mathrm{O}_{2} \text {-Dependent Lung Disease, } \\
\text { DM, OSA }\end{array}$ & blepharoplasty & 53.7 & Cormack II \\
\hline 40 & $\begin{array}{l}\text { acute alcoholic pancreatitis- } \mathrm{pH} 7.14 \text {, lactate } \\
7.3, \mathrm{Cr} 2 . \mathrm{I} \text {, on high-dose norepinephrine and } \\
\text { I5L NBR with } \mathrm{SpO}_{2} 89 \%\end{array}$ & exploratory laparotomy & 27.5 & Cormack I \\
\hline 66 & $\begin{array}{l}\text { concern for retropharyngeal abscess with } \\
\text { stridor- arrived in ED on BiPAP II/5, lethargic } \\
\text { and with SpO2 of } 87 \%\end{array}$ & awake $\mathrm{FOI}$ in the ED & 25.6 & Cormack I \\
\hline 38 & $\begin{array}{l}\text { super morbid obesity, HTN, A.fib, CKD, OSA } \\
\text { in acute respiratory failure from sepsis }\end{array}$ & $\begin{array}{l}\text { awake FOI for perineal } \\
\text { abscess I\&D }\end{array}$ & 95.5 & Cormack II \\
\hline \multicolumn{5}{|c|}{ Maintenance of Anesthesia } \\
\hline 82 & $\begin{array}{l}\text { ICM with EF } 20 \% \text {, OSA, COPD, DM, laryngeal } \\
\text { cancer presenting with severe obstruction and } \\
\text { stridor }\end{array}$ & awake tracheostomy & 33.3 & lowest intraop $\mathrm{SpO}_{2} 100 \%$ \\
\hline 67 & severe spinal stenosis requiring awake $\mathrm{FOI}$ & $\begin{array}{l}\text { single lung transplant- SL } \\
\text { to DL-ETT exchange }\end{array}$ & 32 & lowest intraop $\mathrm{SpO}_{2} 100 \%$ \\
\hline 56 & $\begin{array}{l}\text { HTN, obesity and necrotizing fasciitis of the } \\
\text { lower extremity }\end{array}$ & I\&D lower extremity & 49.2 & lowest intraop $\mathrm{SpO}_{2} 95 \%$ \\
\hline \multicolumn{5}{|c|}{ Postoperative Management } \\
\hline 53 & $\begin{array}{l}\text { OSA, history of cardiogenic shock s/p recent } \\
\text { LVAD placement }\end{array}$ & I\&D sacral wound & 42.4 & indication- hypoxia \\
\hline 71 & history SCC mandible s/p multiple FF revisions & I\&D FF & 23 & indication- hypoxia with FM \\
\hline 77 & severe OSA, COPD on home $\mathrm{O}_{2}$ & $\begin{array}{l}\text { partial glossectomy with } \\
\text { neck dissection }\end{array}$ & 40.4 & indication: patient comfort \\
\hline
\end{tabular}

s/p, status post; LVAD, left ventricular assist device; COPD, chronic obstructive pulmonary disease; ED, emergency department; I\&D, incision and drainage; SCC, squamous cell carcinoma; FF, free flap; ICM, ischemic cardiomyopathy; EF, ejection fraction; DM, diabetes mellitus; HTN, hypertension; SL-ETT, single lumen endotracheal tube; DL-ETT, double lumen endotracheal tube; $\mathrm{HFpEF}$, heart failure with preserved ejection fraction; $\mathrm{SpO}_{2}$, oxygen saturation; NRB, non-breather face mask; FM, face mask; A fib, atrial fibrillation; CKD, chronic kidney disease

Table 1 (section Maintenance of Anesthesia) illustrates key cases where HFNC was felt to provide safer oxygen therapy during maintenance of anesthesia compared to conventional oxygen therapies in a subset of medically challenging patients. Although these individuals were vulnerable to hypoxia, they all maintained close to $100 \%$ saturations $\left(\mathrm{SpO}_{2}\right)$. A cardiac anesthesiologist caring for the patient undergoing a single lung transplant commented: "We had trouble exchanging the endotracheal tube, it took a couple of minutes. These patients often do not tolerate regular intubation without hypoxemia. However, during the difficult and dangerous exchange (with venous-venous ECMO on standby), the patient remained with $100 \% \mathrm{SpO}_{2}$ ".

There were two reported safety concerns with intraoperative use: difficulty obtaining end-tidal $\mathrm{CO}_{2}$ monitoring and potential fire hazard. End-tidal $\mathrm{CO}_{2}$ monitoring was generally overcome by placing a standard face mask with capnography monitoring capability over the patient's face. Although there was some concern for fire potential, no fires were reported, and providers were reminded to avoid $\mathrm{FiO}_{2}>$ $30 \%$ in select cases. 


\section{Postoperative management: $\mathrm{n}=\mathbf{2 3}$}

HFNC therapy was used in the postoperative management of 23 patients. It was most often indicated to treat hypoxia, defined as $\mathrm{SpO}_{2}$ less than $95 \%$ in patients with comorbidities such as OSA, morbid obesity, and COPD not responding to face mask therapy. HFNC was also used in several patients who were agitated with a face mask, most often following laryngeal or neck surgery. These patients appeared more comfortable with HFNC. In all but one patient, HFNC was weaned to standard therapy by the time they left the PACU.

We also coordinated the care of several living-donor liver transplant recipients and extubated them directly to HFNC therapy in the OR, with continuation of this therapy in the transplant ICU for the duration of postoperative day 0 . In this "fast-track extubation pathway," patients were safely transitioned to a regular nasal cannula by postoperative day 1 and none required reintubation.

Table 1 (section Postoperative Management) describes key cases where HFNC was felt to deliver oxygen therapy more effectively during the postoperative care of our patients compared to conventional therapies. These patients were vulnerable to hypoxia and desaturation during their postoperative care; however, all improved with HFNC.

\section{Anesthesia Provider Feedback}

Anesthesiologists overwhelmingly reported that HFNC therapy improved the safety profile of their anesthetic care in situations where they expected desaturations or airway obstruction. We received several comments such as "HFNC really saved me in that case," or "HFNC prevented me from having to reintubate my patient."

\section{Discussion}

Traditionally, oxygen is delivered via low-flow system such as nasal cannula and facemask or high-flow systems such as nonrebreathers and Venturi masks, but all these devices fail to deliver sufficiently humidified oxygen at an accurate $\mathrm{FiO}_{2}$ over the range of flow rates. HFNC can overcome the shortcomings of conventional oxygen delivery devices and is very well-tolerated by the patients. It can deliver a wide range of $\mathrm{FiO}_{2}$ from $21 \%$ to $100 \%$ and flexible flow up to $70 \mathrm{lpm}$ and is still quite tolerable by patients. The advantage of the HFNC is that it can insufflate oxygen, even when a patient become apneic, preventing or delaying the onset of hypoxia and giving the anesthesia provider extra minutes to manage the airway. The washout effect of high oxygen flow on the upper airway dead space will certainly improve ventilation, as well as serve as a great vehicle to move carbon dioxide, ameliorating the hypercarboxia that may be associated with apneic oxygenation. Many studies have demonstrated that $\mathrm{HCNC}$ can generate positive nasopharyngeal airway pressure at the end of exhalation (PEEP), which can potentially decrease the work of breathing and improve alveolar ventilation. ${ }^{12}$

There are few possible contraindications to HFNC that may include sinus surgeries and surgeries of the face and upper airway due to fear of air embolism or barotrauma. The issue of using HFNC in close vicinity of laser or electrocautery was raised by our anesthesia team during neck and/or face procedures that are performed under intravenous sedation; the solution was to use low $\mathrm{FiO}_{2}$ (even at room air concentration) and at a moderate flow rate, which was very successful in preventing hypoxia and risk of fire.

The disadvantage of HFNC is the inability to monitor end-tidal $\mathrm{CO}_{2}$ as an indication of adequate ventilation, which can be an issue, as it is part of anesthesia standards of care. To comply with the standards of care at our institution, we improvised a technique to help with monitoring end-tidal $\mathrm{CO}_{2}$ - we simply used a face mask and cut the tube connecting to the oxygen source and inserted instead the $\mathrm{CO}_{2}$ sampling catheter and were very successful in getting good end-tidal $\mathrm{CO}_{2}$ tracing on the anesthesia monitor.

Over a ten-month period, HFNC was used in the care of 85 medically challenging patients during induction of general anesthesia, intraoperative management, and postoperative care at a large quaternary-care academic healthcare center. We were successful in integrating the HFNC into the existing anesthesia workflow and provided a tool to help the anesthesia team to use it for certain patients and/or certain procedures to tremendously improve patient care. Anesthesiologists utilizing HFNC felt it improved the safety profile of their anesthetic care in clinical scenarios where difficult airway, rapid desaturation, airway obstruction, or hypoxias were expected. No complications were reported, and since this technology then became available to use daily in our ORs and PACU, all the anesthesia providers became familiar with its applications, indications, and advantages.

\section{Conclusion}

In conclusion, HFNC proved to be a very useful tool in the OR and in the PACU and has the potential to improve patient care during the perioperative period.

\section{Funding}

This work was supported by the UPMC Department of Anesthesiology and Perioperative Medicine. Equipment was provided by Fischer and Paykel.

\section{Conflicts of interest/Financial disclosures}

None.

\section{Implication statement}

The use of high flow nasal cannula (HFNC) therapy can increase the safety profile of anesthetic care in certain situations associated with difficult airway, airway obstruction, and hypoxia. Our case series illustrates the broad applications of HFNC in the perioperative setting.

\section{Author contributions}

Philip C. Carullo, MD: This author helped obtain QI project approval, integrate the technology into the operating room, educate staff on use of the technology, recruit patients, and edit the manuscript.

Dennis P. Phillips, DO and Ezeldeen Abuelkasem, MBBCh, MSc: These authors helped integrate the technology into the operating room, educate staff on use of the technology, collect data, and design manuscript Figure and Table.

Ibtesam Hilmi MBCHB, FRCA: This author helped with study design, patient recruitment, and editing the manuscript.

\section{References}

1. Mcginley BM, Patil SP, Kirkness JP, et al. A Nasal Cannula Can Be Used to Treat Obstructive Sleep Apnea. American Journal of Respiratory and Critical Care Medicine. 2007;176(2):194-200.

2. Parke R, Mcguinness S, Eccleston M. Nasal high-flow therapy delivers low level positive airway pressure. Br J Anaesth. 2009;103(6):886-890. 
3. Hernández G, Vaquero C, Colinas L, et al. Effect of Postextubation High-Flow Nasal Cannula vs Noninvasive Ventilation on Reintubation and Postextubation Respiratory Failure in High-Risk Patients. JAMA. 2016;316(15):1565.

4. Ni Y-N, Luo J, Yu H, et al. Can High-flow Nasal Cannula Reduce the Rate of Endotracheal Intubation in Adult Patients with Acute Respiratory Failure Compared with Conventional Oxygen Therapy and Noninvasive Positive Pressure Ventilation? Chest. 2017;151(4):764-775.

5. Stéphan F, Barrucand B, Petit P, et al. High-Flow Nasal Oxygen vs Noninvasive Positive Airway Pressure in Hypoxemic Patients After Cardiothoracic Surgery: A Randomized Clinical Trial. JAMA. 2015;313(23):2331-2339.

6. Yu Y, Qian X, Liu C, et al. Effect of High-Flow Nasal Cannula versus Conventional Oxygen Therapy for Patients with Thoracoscopic Lobectomy after Extubation. Can Respir J. 2017;2017:1-8.

7. Patel A, Nouraei SAR. Transnasal Humidified Rapid-Insufflation Ventilatory Exchange (THRIVE): a physiological method of increasing apnoea time in patients with difficult airways. Anaesthesia. 2014;70(3):323-329.
8. Myatra S, Shah A, Kundra P, et al. All India Difficult Airway Association 2016 guidelines for the management of unanticipated difficult tracheal intubation in adults. Indian Journal of Anaesthesia. 2016;60(12):885.

9. Schumann R, Natov NS, Rocuts-Martinez KA, et al. High-flow nasal oxygen availability for sedation decreases the use of general anesthesia during endoscopic retrograde cholangiopancreatography and endoscopic ultrasound. World J Gastroenterol. 2016;22(47):10398.

10. Tam K, Jeffery C, Sung CK. Surgical Management of Supraglottic Stenosis Using Intubationless Optiflow. Ann Otol Rhinol Laryngol. 2017;126(9):669-672.

11. Lee CC, Perez O, Farooqi FI, et al. Use of high-flow nasal cannula in obese patients receiving colonoscopy under intravenous propofol sedation: A case series. Respir Med Case Rep. 2018;23:118-121.

12. Perke RL, McGuiness SP. Pressure delivered by nasal high flow oxygen during all phase of the respiratory cycle. Respir Care. 2011;56:1151. 